\title{
Seizure activity results in calcium- and mitochondria- independent ROS production via NADPH and xanthine oxidase activation
}

\author{
S Kovac ${ }^{\star, 1,2}$, A-M Domijan ${ }^{3}$, MC Walker $^{1,4}$ and AY Abramov ${ }^{1,4}$
}

Seizure activity has been proposed to result in the generation of reactive oxygen species (ROS), which then contribute to seizureinduced neuronal damage and eventually cell death. Although the mechanisms of seizure-induced ROS generation are unclear, mitochondria and cellular calcium overload have been proposed to have a crucial role. We aim to determine the sources of seizureinduced ROS and their contribution to seizure-induced cell death. Using live cell imaging techniques in glioneuronal cultures, we show that prolonged seizure-like activity increases ROS production in an NMDA receptor-dependent manner. Unexpectedly, however, mitochondria did not contribute to ROS production during seizure-like activity. ROS were generated primarily by NADPH oxidase and later by xanthine oxidase $(\mathrm{XO})$ activity in a calcium-independent manner. This calcium-independent neuronal ROS production was accompanied by an increase in intracellular $\left[\mathrm{Na}^{+}\right]$through NMDA receptor activation. Inhibition of NADPH or XO markedly reduced seizure-like activity-induced neuronal apoptosis. These findings demonstrate a critical role for ROS in seizureinduced neuronal cell death and identify novel therapeutic targets.

Cell Death and Disease (2014) 5, e1442; doi:10.1038/cddis.2014.390; published online 2 October 2014

Reactive oxygen species (ROS) contribute to neuronal damage and have been linked to excitotoxicity. ${ }^{1-4}$ An increase in ROS generation has also been identified in acute neurologic disease such as stroke, ${ }^{5,6}$ and recent evidence indicates that this may contribute to neuronal damage in seizures and epilepsy. ${ }^{7-10}$ However, ROS measurements during seizurelike activity were predominantly performed in homogenates, extracellular fluids or brain regions with no clear demonstration of whether the ROS were of neuronal origin. ${ }^{9,11,12}$ Moreover, these studies lacked the necessary temporal resolution to determine accurately the evolution of ROS generation during and after prolonged seizure activity. Such obstacles can be overcome by live cell imaging of ROS, which has emerged as a powerful tool to study disease mechanisms. ${ }^{13}$

If seizure activity induces ROS production in neurons, then a critical question is which sources of ROS production are triggered by such activity. Previous studies have suggested that mitochondria are the primary source of ROS generation in seizure models. ${ }^{8,14}$ However, there are alternative sources of ROS, in particular the enzymes NADPH oxidase and xanthine oxidase (XO). How these contribute to excitotoxicity during seizure activity is uncertain. That these enzymes may have an important role in seizure-induced ROS generation is suggested by two observations: (1) NMDA receptors have a pivotal role in seizure-induced neuronal damage ${ }^{15}$ and (2) direct pharmacologic activation of NMDA receptors can activate NADPH oxidase, increasing free radical production and consequently neuronal death., ${ }^{5,16,17}$ There is also burgeoning evidence of a role for NADPH oxidase activation in chronic brain pathology secondary to psychosocial stress, which leads to the development of neuropathologic alterations, and also in neurodegenerative disease. ${ }^{18,19}$

Acute activation of NADPH oxidase in neurons has mainly been shown after direct pharmacologic activation of NMDA receptors via exposure to high levels of NMDA and this activation is calcium-dependent. ${ }^{16,17}$ More recently, activation of NADPH oxidase has been shown during seizure activity. ${ }^{9,20}$ These pathways also involved NMDA receptor activation and upregulation of NMDA receptor subunits NR1 and NR2B. Nonetheless, these studies used chemoconvulsant epilepsy models, which, in themselves, may have an impact on ROS generation. The mechanisms and relevance of activation of NADPH oxidase during seizure activity independent of chemoconvulsants is unclear, especially given the presence of alternative sources of ROS production. Moreover, XO may also represent a major potential source of ROS during periods of increased metabolism, such as that occuring during seizures. We have therefore asked whether NMDA receptor activation has a role in seizure-induced ROS production and which sources and mechanisms of ROS production are involved in its time course during seizure-like activity.

Here, we demonstrate increased ROS generation during seizure-like activity. This is activity-dependent, but it is maintained by a $\mathrm{Ca}^{2+}$-independent pathway involving the

\footnotetext{
${ }^{1}$ UCL Institute of Neurology, University College London, Queen Square, London WC1N 3BG, UK; ${ }^{2}$ Department of Neurology, University of Muenster, Muenster 48149, Germany and ${ }^{3}$ Faculty of Pharmacy and Biochemistry, University of Zagreb, Zagreb 10000, Croatia

*Corresponding author: S Kovac or AY Abramov, UCL Institute of Neurology, University College London, Queen Square, London WC1N 3BG, UK. Tel: +44 (0)20 78374062 ; Fax: +44 (0)20 7837 5069; E-mail: stjepana.kovac.09@ucl.ac.uk or a.abramov@ucl.ac.uk

${ }^{4}$ These authors contributed equally to this work.

Abbreviations: ROS, reactive oxygen species; XO, xanthine oxidase; PKC, protein kinase C; PMCA, plasma membrane Ca2 + ATPase; NMDAR, NMDA receptor; SOD, superoxide dismutase; HEt, hydroethidine; H2DFFDA, 5- and 6- carboxy-2',7'-dichlorofluorescein; NOX2, NADPH oxidase 2; 4-AP, 4-aminopyridine Received 25.2.14; revised 14.7.14; accepted 15.7.14; Edited by A Verkhratsky
} 
activation of NMDA receptors, NADPH oxidase and $\mathrm{XO}$ at a later phase. Blocking NADPH oxidase and $\mathrm{XO}$ prevented seizure-induced neuronal cell death in vitro. We thus provide compelling evidence that these ROS-generating pathways are appropriate targets for preventing neuronal death in seizures.

\section{Results}

ROS generation and lipid peroxidation in vitro in the low magnesium model. To determine the mechanisms and sources of ROS generation, and their exact temporal relationship during the initial phase of network hyperexcitability, the well-established low magnesium culture model of seizure-like activity was used. ${ }^{21,22}$ We performed live cell imaging experiments in rat neocortical glioneuronal cocultures (days in vitro 12-21; Figure 1a). In keeping with previous reports, we found that seizure-like activity in the low magnesium model induced oscillatory increases in intracellular $\mathrm{Ca}^{2+}$, monitored with fura-2 (Figure $1 \mathrm{~b}$ and Supplementary Video 1), ${ }^{23}$ coinciding with rapid burst firing of neurons, as has been shown previously. ${ }^{24}$ These changes resulted in an immediate and significant increase in the rate of ROS production in neurons (Figure 1c), while the rate of ROS production in control cultures (neurons treated with aCSF) was no different from baseline (Figure 1c). The response was not homogeneous in all cells. Based on a qualitative analysis of the rates of dihydroethidium (HEt) fluorescence, we could distinguish two phases: $\sim 2$ min and 10 min after low magnesium exposure, and these time windows were thus used for analysis (Figure 1c). There was a statistically significant difference of ROS production between the low magnesium and control (repeated-measures ANOVA; $F(1,223)=56.4 ; P<0.001)$. There was also a significant interaction between time and group $(F \quad(1,223)=26.4$, $P<0.001)$, indicating that as time progressed the difference between the groups became significantly larger (Figure 1d). Rates of ROS production measured after 2 min in neurons exposed to low magnesium $(n=188)$ were almost two times that of control neurons ( $n=41 ; 166 \pm 7 \%$ versus $85 \pm 2 \%$ ). There was a further increase in the rate of ROS production in neurons observed $10 \mathrm{~min}$ after omission of magnesium; ROS production in these neurons was almost fourfold greater than that seen in neurons under control conditions ( $n=125 ; \quad 337 \pm 17 \%$ versus $83 \pm 7 \%$ ). To confirm ROS production using different approaches or indicators, we conducted identical experiments and obtained similar results using 530/380 ratio or measurements of 5- and 6- carboxy-2',7'-dichlorofluorescein $\left(\mathrm{H}_{2} \mathrm{DFFDA}\right)$ fluorescence (Figures 1e and f).

ROS are known to induce oxidative degradation of lipids (lipid peroxidation). We therefore determined lipid peroxidation in the low magnesium culture model of seizure-like activity using C11-BODIPY, an indicator of lipid peroxidation. The rate of lipid peroxidation increased $\sim 2$-fold as a result of lowmagnesium-induced seizure-like activity $(n=32 ; P<0.001$; paired $t$-test; Figures $1 \mathrm{~g}$ and $\mathrm{h}$ ).

Omission of magnesium from the superfusion solution induces oscillatory increases in neuronal $\left[\mathrm{Ca}^{2+}\right]_{\mathrm{c}}$ (Kovacs et al.; ${ }^{25}$ Figure 1b). We hypothesized that increases in neuronal ROS reflect the activity status of neurons. Simultaneous $\left[\mathrm{Ca}^{2+}\right]_{\mathrm{c}}$ and ROS measurements indicated that the neuronal ROS production coincided with the $\mathrm{Ca}^{2+}$ signal and was greater in neurons with larger and more sustained $\mathrm{Ca}^{2+}$ oscillations (Supplementary Figures $1 \mathrm{~A}$ and B).

To confirm that increases in ROS production were not a peculiarity of the low magnesium model of seizure-like activity, we reproduced our findings using another in vitro epilepsy model. A well-established convulsant, ${ }^{23,26,27}$ 4-aminopyridine (4-AP), also induced $a \sim 1.5$ - to 2 -fold increase in ROS production compared with baseline at both time points ( $n=126$; $2 \mathrm{~min}: 195 \pm 11 \%$; $10 \mathrm{~min}$ : $168.5 \pm 10 \%$; repeatmeasures ANOVA; F $(1,125)=464.6 ; P<0.001$; Figures $2 a$ and $b$ ), confirming that the increase in ROS production is not model-specific. In addition, we measured ROS production after activation with low concentrations of glutamate, which has been used as an in vitro model of epilepsy, ${ }^{28}$ yielding similar results ( $n=76$; 2 min: $262 \pm 18 \% 10 \min : 179 \pm 12 \%$ (glutamate); repeat-measures ANOVA; F $(1,75)=291.0$; $P<0.001$; Figure 2c).

Unfortunately, we could not measure ROS with genetically encoded ROS probes such as HyPer-3, as treatment with low magnesium induced acidification; this was shown with the $\mathrm{H}_{2} \mathrm{O}_{2}$-insensitive version of HyPer-3, HyPer-C199S $(n=6$; Supplementary Figure 2E). Changes in $\mathrm{pH}$ were confirmed using SNARF, which also demonstrated neuronal acidification during low-magnesium-induced seizure-like activity $(n=29$; Supplementary Figures 2A-D).

Contribution of NMDA receptors and extracellular $\mathrm{Ca}^{2+}$ to low-magnesium-induced ROS generation in neurons in vitro. Low-magnesium-induced $\mathrm{Ca}^{2+}$ oscillations, representing seizure-like activity, have been shown to be NMDA receptor-sensitive. ${ }^{29}$ Blocking the NMDA receptor with NMDA

Figure 1 Seizure-like activity in the low magnesium model of epilepsy induces ROS production in primary cortical neurons. (a) Phase-contrast image showing rodent glioneuronal cultures in vitro and (b) seizure-like activity induced in neurons in the low magnesium model, as indicated by repetitive $\mathrm{Ca}^{2+}$ oscillations measured with the calcium indicator fura-2 (see also Supplementary Video 1). Each trace in (b) represents a single neuron. Note that $\mathrm{Ca}^{2+}$ transients in neurons are synchronous and rhythmic characteristic of seizure-like activity. (c) HEt fluorescence measurements in neurons (mean \pm S.E.M.) from a representative experiment showing low-magnesium-induced increases in the HEt fluorescence in neurons exposed to low magnesium treatment (solid circle) and stable rates of HEt fluorescence indicating stable ROS generation rates in neurons treated with aCSF only (control; unfilled circle). The vertical dashed line indicates replacement of the solution to either low magnesium (solid circle) or aCSF (unfilled circle) after a baseline with aCSF. Bars indicate representatives periods that were taken to analyse the rate of ROS generation. (d) Mean rates of ROS generation at the representative time points in neurons undergoing low magnesium treatment and neurons treated with aCSF only with the basal rate in neurons set as $100 \%$. ROS generation in neurons as measured with (e) ratiometric Het fluorescence imaging and (f) using $\mathrm{H}_{2}$ DFFDA. Traces in (e) and (f) show fluorescence measurements from single representative neurons (circles). Bottom traces (triangles) in these two panels indicate the differentiated trace. (g) Lipid peroxidation in neurons during low-magnesium-induced seizure-like activity as measured with C11BODIPY ratio in a representative experiment (mean \pm S.E.M.; upper trace). The bottom traces (triangles) indicates the differentiated mean C11-BODIPY fluorescence of the trace represented above. (h) Histogram summarizing rates of lipid peroxidation during baseline (initial rate) and during low magnesium seizure-like activity (low Mg ${ }^{2+}$ ). Error bars indicate S.E.M. ${ }^{* \star} P<0.001$ and ${ }^{* \star} P<0.01$ 
receptor antagonists (APV: $25 \mu \mathrm{M} ; n=30$ or MK801: $10 \mu \mathrm{M}$; $n=56)$ or the NR2B subunit specific antagonist ifenprodil $(10 \mu \mathrm{M} ; n=73)$ abolished the low-magnesium-induced $\mathrm{Ca}^{2+}$ signal (Figures $2 \mathrm{~d}-\mathrm{f}$ ). $\mathrm{Ca}^{2+}$-free low magnesium solution also abolished the low-magnesium-induced $\mathrm{Ca}^{2+}$ signal, suggesting that external calcium is pivotal in maintaining low-

a
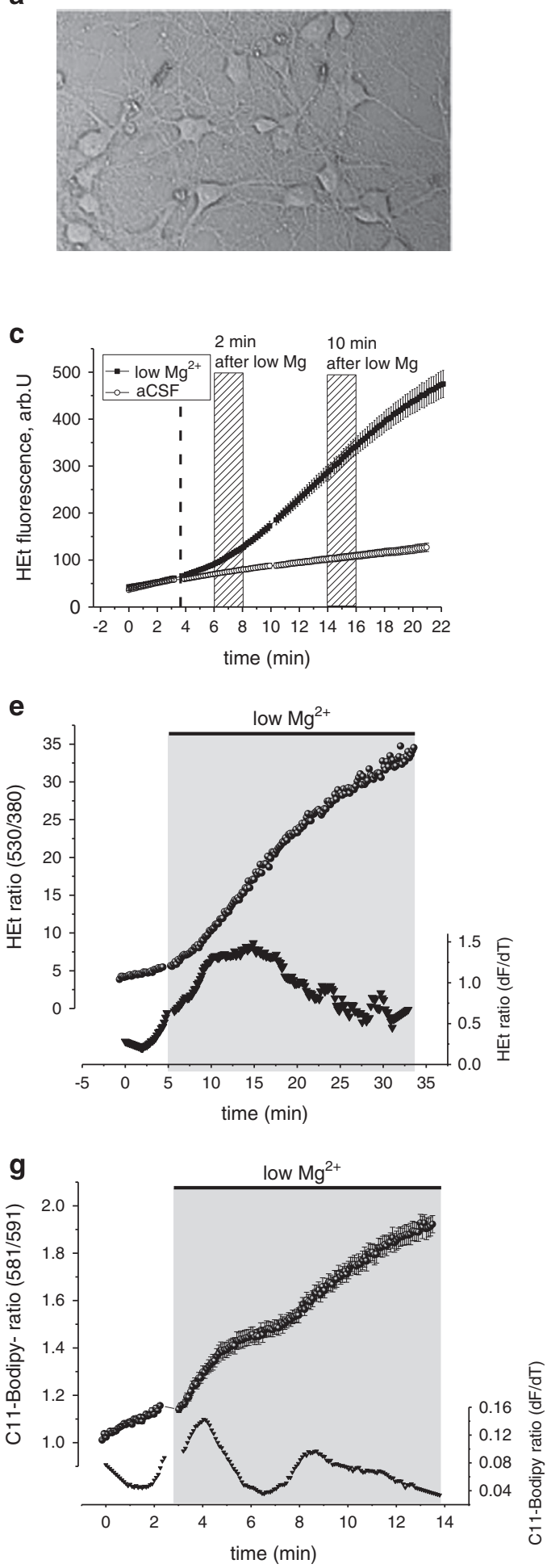

magnesium-induced $\mathrm{Ca}^{2+}$ oscillations in neurons $(n=37$; Figure 2g).

Given that the $\left[\mathrm{Ca}^{2+}\right]_{\mathrm{c}}$ oscillations in the low magnesium model are dependent on NMDA receptors and external $\mathrm{Ca}^{2+}$ entry, we determined how each of these factors contributed to ROS generation observed
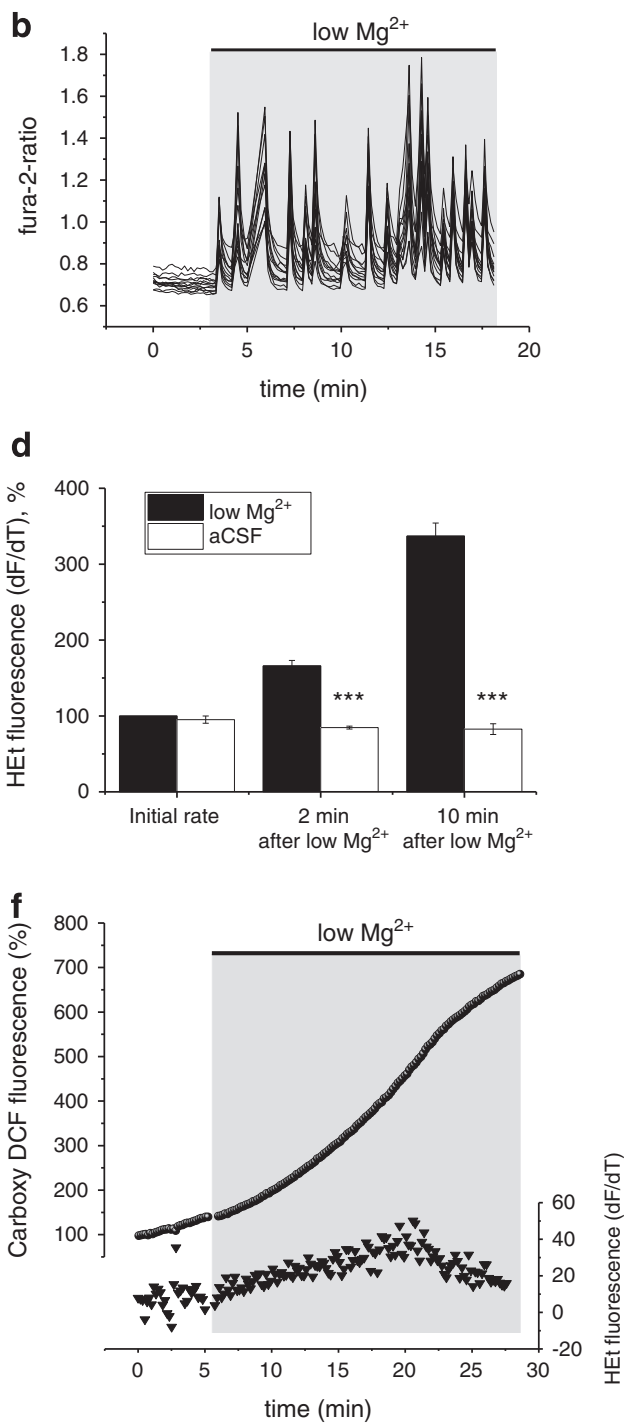

h

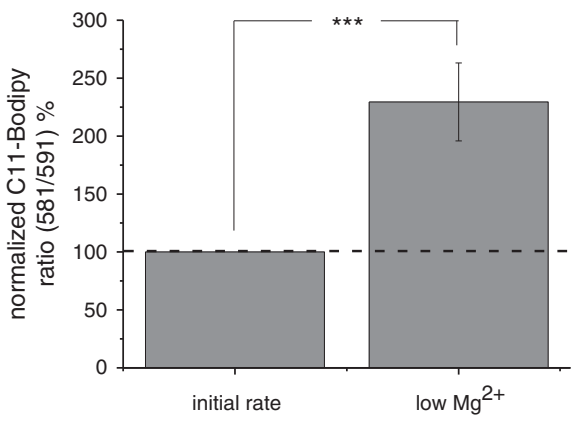



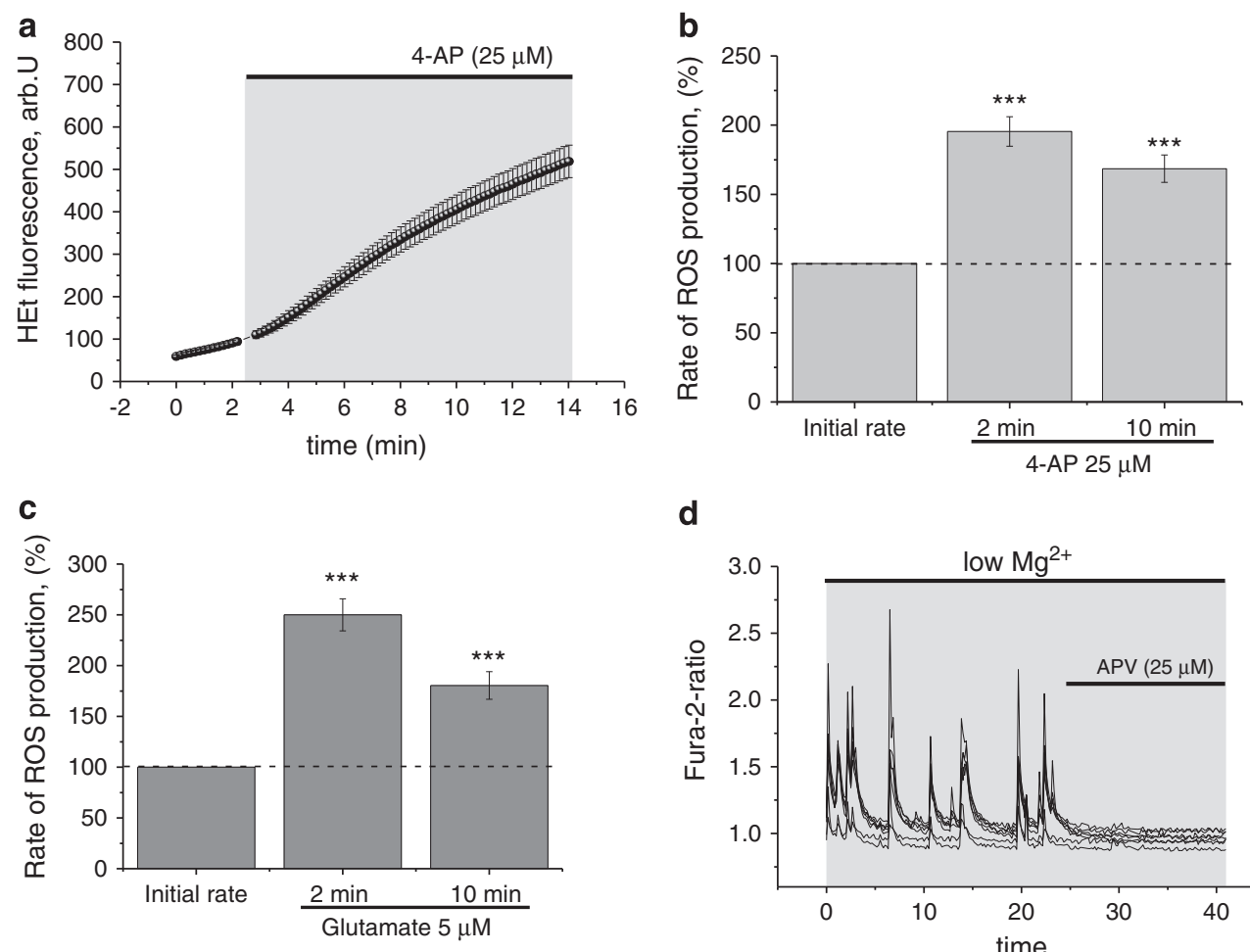

d
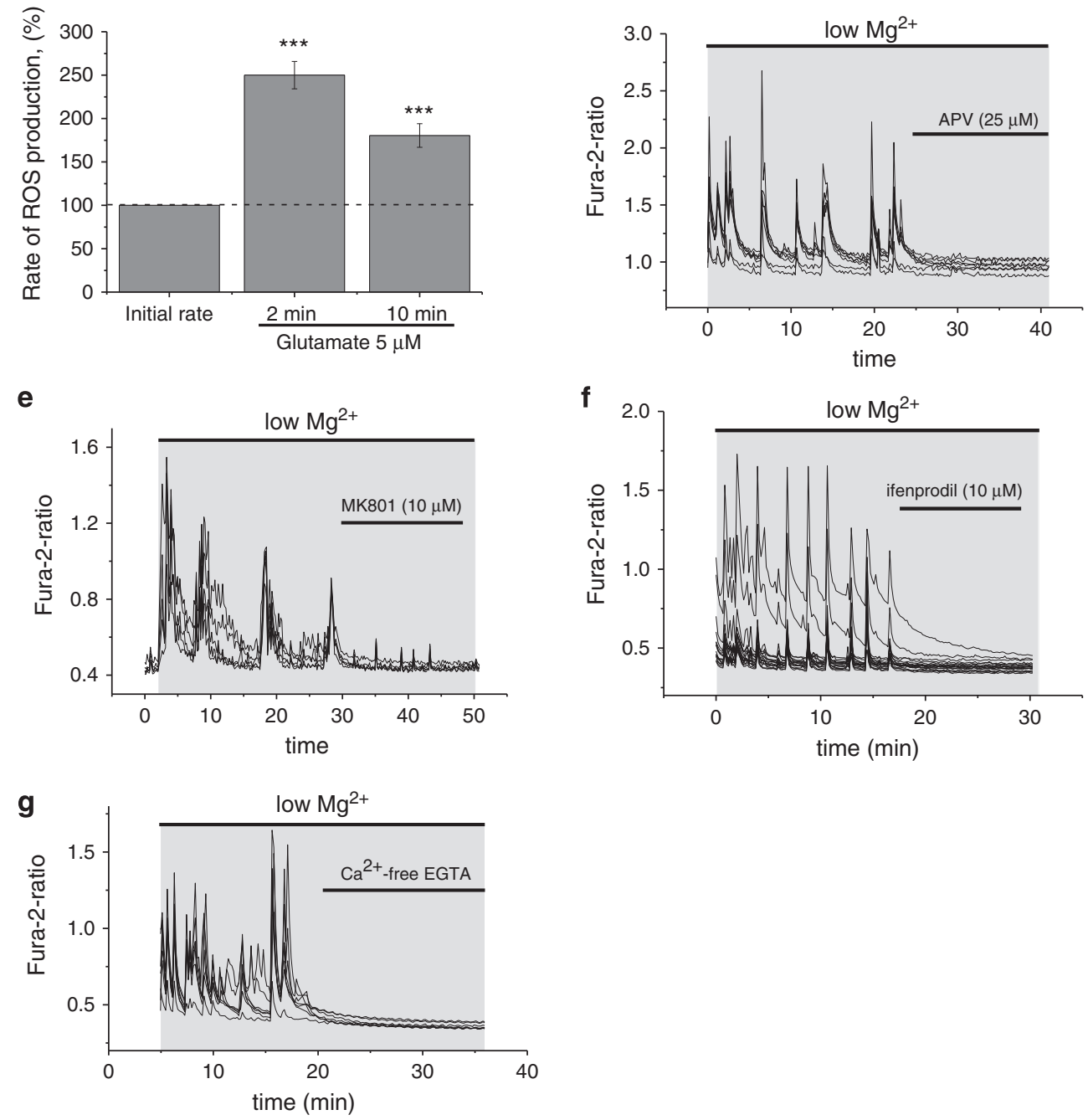

Figure 2 ROS changes in neurons during 4-AP and glutamate-induced epileptiform activity. (a) HEt fluorescence measurements in neurons (mean \pm S.E.M.) from a representative experiment showing 4-AP induced increases in the HEt fluorescence in neurons (solid circle). (b+c) Histogram summarizing ROS changes in neurons in (b) the 4-AP model and in (c) glutamate model of seizure-like activity (mean \pm S.E.M.). ${ }^{* \star *} P<0.001$. NMDA receptor sensitivity of low-magnesium-induced $\mathrm{Ca}^{2+}$ signals in neurons. Blocking the NMDA receptor with (d) APV $(25 \mu \mathrm{M})$, (e) MK801 (10 $\mu \mathrm{M})$, (f) ifenprodil $(10 \mu \mathrm{M})$, an antagonist of NR2B subunit of NMDA receptor, or $(\mathbf{g})$ omitting Ca ${ }^{2+}$ and adding the $\mathrm{Ca}^{2+}$ chelator (+EGTA $0.5 \mathrm{mM}$ ) to the external solution abolished the established low-magnesium-induced $\mathrm{Ca}^{2+}$ signal in neurons. Low-magnesium-induced $\mathrm{Ca}^{2+}$ signals in neurons are presented as fura-2 ratios. Each trace represents a single neuron

in this model. We measured HEt fluorescence in the presence of different NMDA receptor blockers (MK801, APV and ifenprodil) or $\mathrm{Ca}^{2+}$-free low magnesium aCSF and compared rates of ROS production with the rates obtained with low magnesium treatment alone (Figures $3 a$ and $b$ ).

There was a statistically significant difference in the rate of ROS production between the groups (repeat-measures 

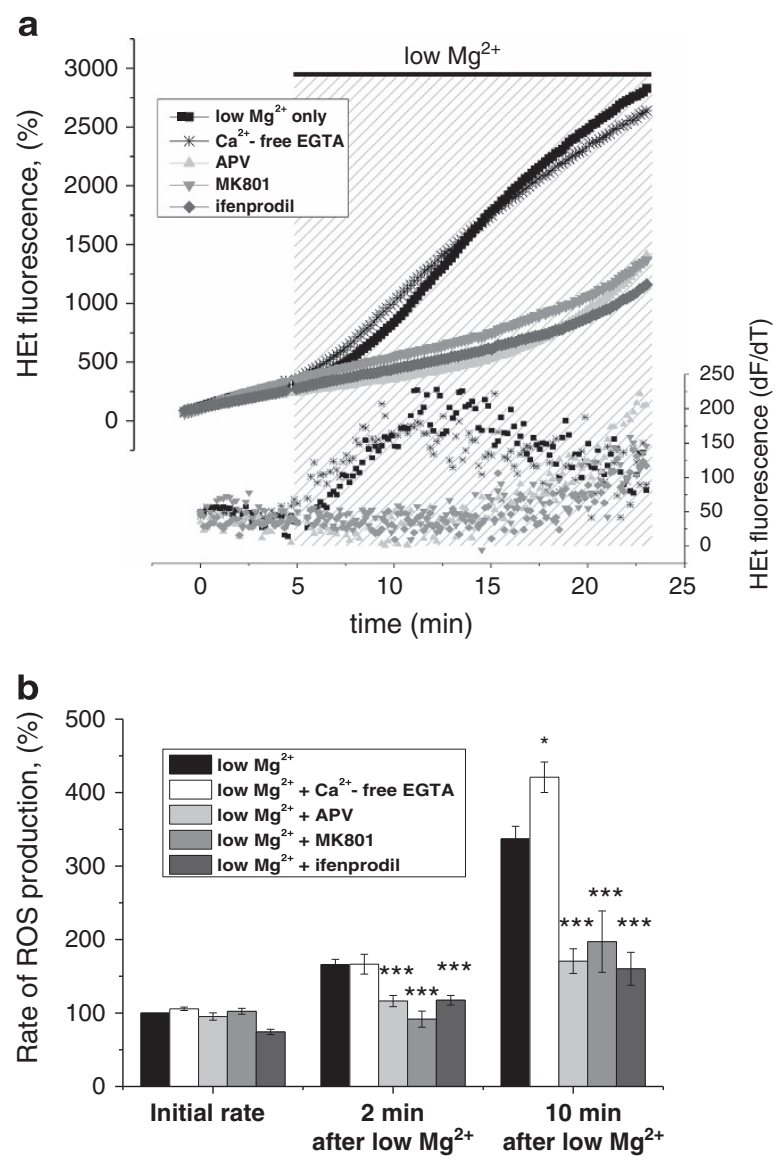

Figure $3 \mathrm{Ca}^{2+}$ and NMDA receptor involvement in low-magnesium-induced ROS generation in neurons. (a) The upper panel represents HEt fluorescence in single, representative neuron during low magnesium seizure-like activity, whereas scatter plot in the bottom panel represents the differentiated signal as shown in the upper panel reflecting the rate of ROS generation. Antagonizing NMDA receptors with APV $(25 \mu \mathrm{M})$, MK801 $(10 \mu \mathrm{M})$ or with antagonist of the NRB2 subunit of NMDA receptor ifenprodil $(10 \mu \mathrm{M})$ abolished the low-magnesium-induced increase in ROS generation. Note that neuronal ROS generation was not abolished in low $\mathrm{Mg}^{2+}$ $\mathrm{Ca}^{2+}$-free aCSF. (b) Histogram summarizing mean \pm S.E.M. rates of ROS generation at 2 and 10 min after omission of magnesium from the extracellular solution for the different treatment groups. ${ }^{*} P<0.05$ and ${ }^{* \star *} P<0.001$

ANOVA; $F(4,405)=19.5 ; P<0.001)$. There was also a significant interaction between time and group $F$ $(4,405)=11.8, P<0.001)$, indicating that as time progressed the difference between the groups became significantly larger (Figure 3b). Blocking the NMDA receptor in neurons with APV $(n=56 ; 25 \mu \mathrm{M})$, MK801 $(n=65 ; 10 \mu \mathrm{M})$ and ifenprodil $(n=52$; $10 \mu \mathrm{M}$ ) in low magnesium conditions significantly reduced the rate of ROS production both $2 \mathrm{~min}$ and $10 \mathrm{~min}$ after omission of magnesium when compared with control (Figures $3 a$ and b). Despite blocking low-magnesium-induced $\left[\mathrm{Ca}^{2+}\right]_{\mathrm{c}}$ signals in neurons (Figure $2 \mathrm{~g}$ ), $\mathrm{Ca}^{2+}$-free low magnesium aCSF had no suppressive effect on neuronal low-magnesium-induced ROS formation (Figures $3 a$ and b). Neuronal rates of ROS generation in $\mathrm{Ca}^{2+}$-free low magnesium did not differ significantly from neuronal rates of ROS generation in low magnesium conditions at 2 min (Figure $3 b$ ) and ROS production at $10 \mathrm{~min}$ was even slightly higher in neurons exposed to $\mathrm{Ca}^{2+}$-free low magnesium compared with low magnesium only, indicating, surprisingly, that seizure-induced ROS generation is not dependent on $\mathrm{Ca}^{2+}$ influx.

As ROS generation is NMDA receptor dependent when $\mathrm{Ca}^{2+}$ is present, we hypothesized that ROS generation in $\mathrm{Ca}^{2+}$-free low magnesium aCSF would also depend on NMDA receptor activation.

There was a statistically significant difference of ROS production between neurons treated with APV $(25 \mu \mathrm{M}$; $n=25)$ in $\mathrm{Ca}^{2+}$-free low magnesium aCSF and neurons treated with $\mathrm{Ca}^{2+}$-free low magnesium aCSF, only (repeatmeasures ANOVA; $F(1,111)=24.0 ; P<0.001$; Figures $4 a$ and $b$ ). There was a statistically significant interaction between the effects of group ( $\mathrm{APV}+\mathrm{Ca}^{2+}$-free low magnesium aCSF versus $\mathrm{Ca}^{2+}$-free low magnesium aCSF) and time point measured on ROS production, indicating that as time progressed the difference between the groups became significantly larger $(F(1,111)=6.0 ; P<0.05)$. Blocking NMDA receptors with APV $(25 \mu \mathrm{M} ; n=25)$ significantly reduced the ROS increase in $\mathrm{Ca}^{2+}$-free low magnesium aCSF both 2 and 10 min after low magnesium treatment (Figures $4 a$ and b). Imaging of intracellular sodium with SBFI showed that omission of magnesium from $\mathrm{Ca}^{2+}$-free aCSF induced an immediate rise of intracellular sodium ( $n=87$; Figure $4 c$ ). This intracellular sodium rise was blocked with APV $(n=32$; Figure 4d), indicating that ROS production in $\mathrm{Ca}^{2+}$-free low magnesium conditions can be maintained by a current through the NMDA receptor linked to an increase in intracellular sodium through NMDA receptors (Figures $4 c$ and $d$ ).

Sources of ROS generation in low magnesium model. Recent studies have suggested that excessive pharmacologic NMDA receptor activation predominantly induces ROS production via activation of NADPH oxidase. ${ }^{17}$ Given that low-magnesium-induced ROS generation was NMDA receptor dependent, we asked whether ROS generation can be blocked by inhibiting NADPH oxidase with AEBSF $(20 \mu \mathrm{M})$, a well-established inhibitor of NADPH oxidase activation. ${ }^{30}$ Excessive neuronal activity has been linked to ATP depletion. ${ }^{23}$ Intracellular ATP depletion results in increased adenine formation and consequently an increase in hypoxanthine and xanthine, substrates for $\mathrm{XO} \cdot{ }^{31,32}$ We therefore hypothesized that late phases of ROS generation in the low magnesium model might be due to hypoxanthine and xanthine oxidation by $\mathrm{XO}$, a process that generates hydrogen peroxide. To distinguish between these two pathways, we blocked low-magnesium-induced ROS production using the NADPH oxidase blocker AEBSF or the XO blocker oxypurinol. Both AEBSF and oxypurinol reduced ROS production (Figures $5 a-d$ ). There was a statistically significant difference in the rate of ROS production between the groups (repeatmeasures ANOVA; $F(1,292)=14.2 ; P<0.001$; Figure $5 d$ ). There was a significant interaction between time and group as time progressed; the difference between the groups became significantly larger $(F(1,292)=8.7 ; P<0.001$; Figure $5 \mathrm{~d}$ ). ROS production at 2 and at $10 \mathrm{~min}$ was significantly lower if AEBSF $(n=41 ; 20 \mu \mathrm{M})$ was added to low magnesium aCSF when compared with low-magnesiumtreated neurons only (Figures $5 \mathrm{a}, \mathrm{b}$ and $\mathrm{d}$ ). Inhibition of $\mathrm{XO}$ with oxypurinol $(n=34 ; 20 \mu \mathrm{M})$ abolished the late phase of low-magnesium-induced ROS production ( $>15 \mathrm{~min}$ after 

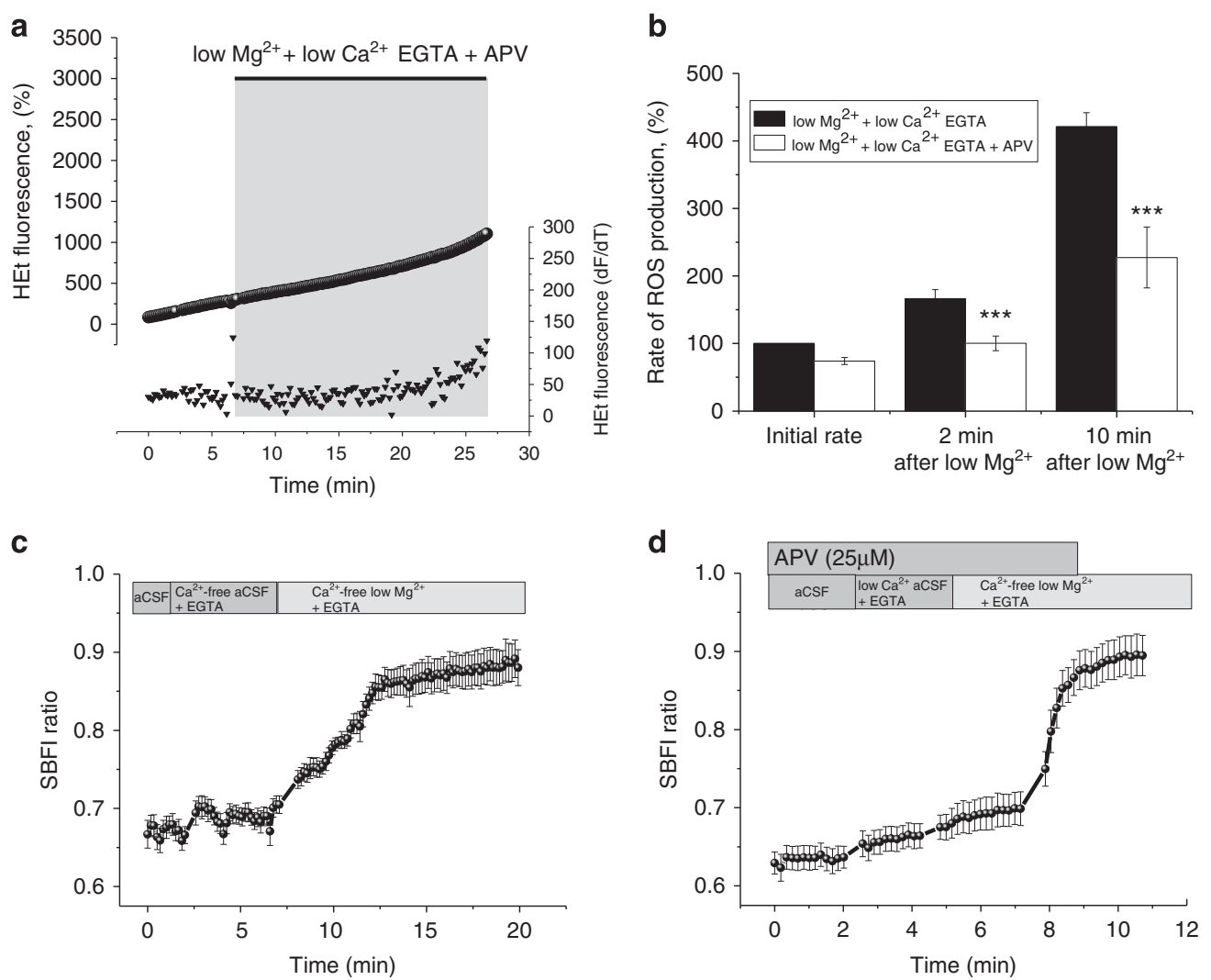

Figure 4 NMDA receptor and sodium current involvement in low magnesium $\mathrm{Ca}^{2+}$-free EGTA induced ROS generation. (a) HEt fluorescence from a representative neuron under low $\mathrm{Mg}^{2+}$ - and $\mathrm{Ca}^{2+}$-free conditions and NMDA receptor antagonism with APV ( $25 \mu \mathrm{M}$; circles). The bottom trace (triangles) shows the HEt signal after differentiation (triangles) reflecting the rate of ROS generation. Note that ROS generation is abolished under these conditions. (b) Histogram summarizing the mean rates of ROS generation in neurons under low $\mathrm{Mg}^{2+}$ - and $\mathrm{Ca}^{2+}$-free conditions (black) compared with low $\mathrm{Mg}^{2+}$ - and $\mathrm{Ca}^{2+}$-free conditions in the presence of APV (25 $\mu \mathrm{M}$; white). (c) Intracellular sodium levels in neurons as measured with SBFI increased after omission of $\mathrm{Mg}^{2+}$ - from $\mathrm{Ca}^{2+}$-free aCSF. (d) This increase can be blocked with the NMDA receptor antagonist APV $(25 \mu \mathrm{M})$. Traces in $(\mathbf{c})$ and $(\mathbf{d})$ show the mean \pm S.E.M. value of the SBFI fluorescent signal measured in all neurons in one representative experiment. ${ }^{* \star *} P<0.001$

oxypurinol application; Figure 5c). These cells showed a high rate of ROS generation in the early phase (Figures $5 \mathrm{c}$ and d). In addition, 30 min preincubation with gp91 ds-tat $(n=53)$, a specific peptide inhibitor of NADPH oxidase assembly, ${ }^{33}$ inhibited ROS production in the low magnesium model of seizure-like activity when compared with gp91 ds-tat scrambled ( $n=43 ; P<0.001 ; t$-test; Figures $5 e$ and f). We next blocked Rac1, an activator of the NOX complex. ${ }^{34}$ Inhibition of Rac1 with NSC-23766 $(n=73)$ prevented seizure-induced ROS increase when compared with low magnesium treatment only $(n=54 ; \quad P<0.001 ; \quad t$-test; Figure 5f).

We failed to capture the secondary increase in ROS and its inhibition with XO by statistical analysis owing to the time differences in the appearance of the secondary ROS generation increase as can be seen by qualitative visual assessment of the raw data (Supplementary Figure 3). Unlike oxygen deprivation, ${ }^{5}$ seizure-like activity is a more subtle and variable dysfunction with neuronal excitotoxicity and cell death occurring at different time points.

There was a statistically significant difference in the rate of ROS production between neurons treated with AEBSF $(20 \mu \mathrm{M})$ in $\mathrm{Ca}^{2+}$-free low magnesium aCSF and neurons treated with $\mathrm{Ca}^{2+}$-free low magnesium aCSF, only (repeatmeasures ANOVA; $F(1,122)=75.1 ; P<0.001)$. There was a significant interaction between time and group, as time progressed the difference between the groups became significantly larger $(F(1,122)=24.5 ; P<0.001$; Figures $6 a$ and b). Blocking NADPH oxidase with AEBSF $(20 \mu \mathrm{M})$ significantly reduced rates of ROS generation 2 and $10 \mathrm{~min}$ after superfusion of low magnesium $\mathrm{Ca}^{2+}$-free aCSF when compared with low magnesium $\mathrm{Ca}^{2+}$-free treatment only $(n=36$; Figures $6 \mathrm{a}$ and b). Similarly, AEBSF blocked ROS production in 4-AP and in response to glutamate ( $n=126,195 \pm 11 \%$ (4-AP) versus $n=8299 \pm 9 \%$ (4-AP and AEBSF) and $n=76 ; 262 \pm 18$ (glutamate) versus $n=70$ $151 \pm 13 \%$ (AEBSF and glutamate); both $P<0.001$; $t$-test; Supplementary Figure 4).

Previous studies have suggested that increased ROS in the low magnesium model are of mitochondrial origin. ${ }^{8,35}$ Comonitoring ROS of mitochondrial origin and intracellular $\mathrm{Ca}^{2+}$ changes in neurons, using the mitochondrial ROS indicator MitoSOX and $\mathrm{Ca}^{2+}$ indicator Fluo-4, showed that in neurons showing low-magnesium-induced $\mathrm{Ca}^{2+}$ oscillations, no increase of ROS of mitochondrial origin could be detected (Figures $7 \mathrm{a}$ and b). We tested rates at different time points. There was a significant change in ROS production between the time points (repeat-measures ANOVA; $F(2,44)=4.1$; $P<0.05$ ). There was a decrease in the rate of ROS generation over time that could be attributed to mitochondrial 
a
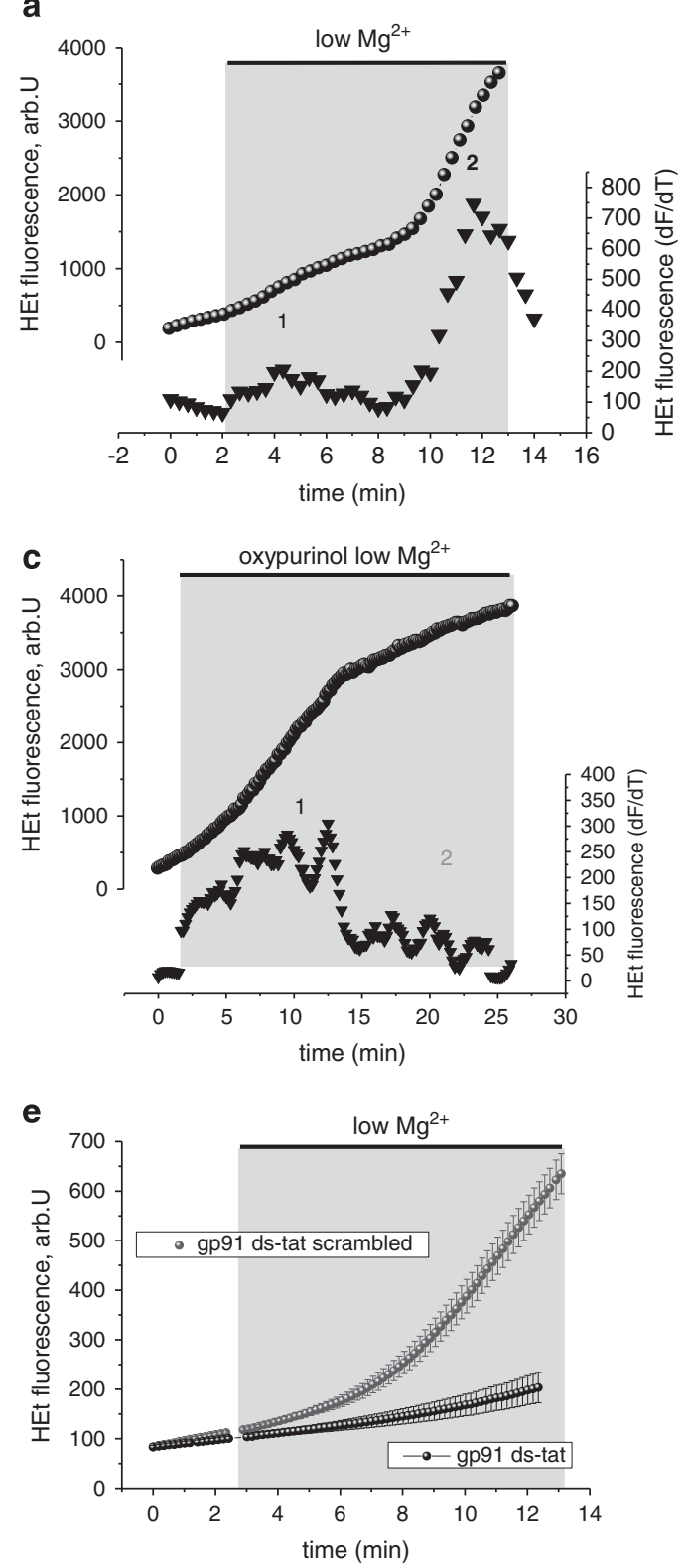

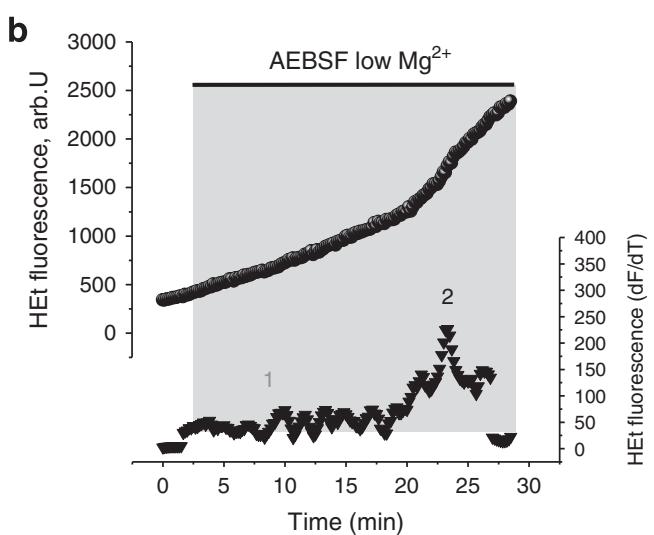

d
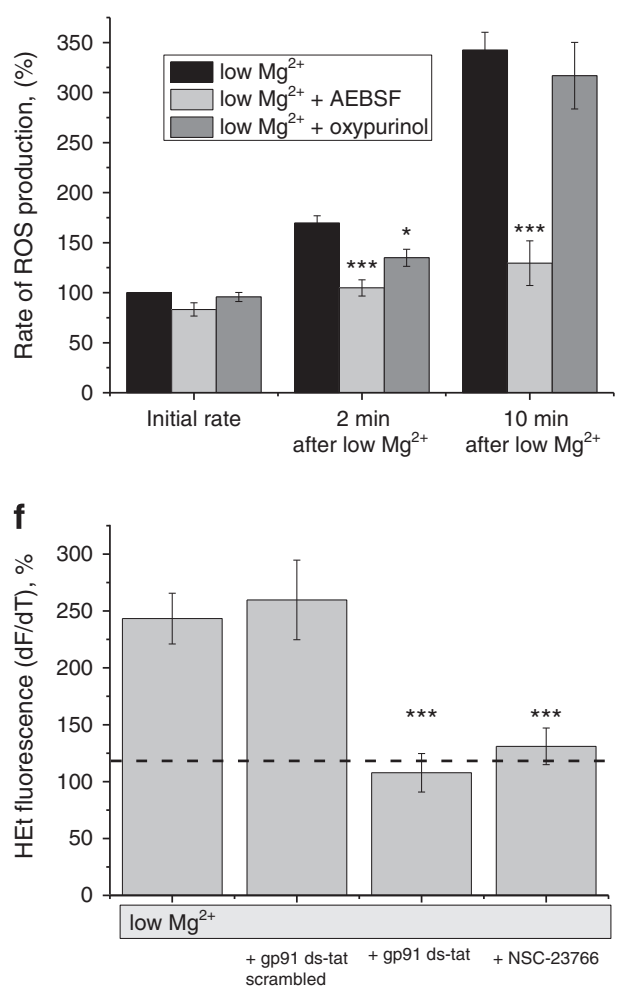

Figure 5 Two distinct phases of ROS generation were seen in the low magnesium model of epilepsy: (a) Two distinct phases of ROS generation were observed after neuronal exposure to low magnesium condition. The bottom trace represents the differentiation of the signal (triangle) and thus reflects the rate of ROS generation in the neuron. The numbers (1 and 2) highlight the key phases of ROS generation in the bottom traces showing the differentiated HEt signal. The key mechanisms of ROS generation were identified by (b) antagonism of the NADPH oxidase with AEBSF $(20 \mu \mathrm{M})$, which abolished phase 1, and (c) XO inhibition with oxypurinol (20 $\mu \mathrm{M})$, which abolished phase 2. Traces in (a-c) show fluorescence measurements from single neurons (circles). (d) Histogram summarizing the impact of NADPH oxidase and XO inhibitors on low-magnesium-induced the ROS generation at different time points. Error bars indicate S.E.M. ${ }^{* \star *} P<0.001$ and ${ }^{*} P<0.05$. Effect of NADPH oxidase inhibitor gp91 ds-tat and Rac1 activator NSC-23766 on ROS production in the low magnesium model of seizure-like activity: (e) Het fluorescence measurements in the low magnesium model after blocking with gp91 ds-tat, an NADPH oxidase assembly inhibitor. Each trace represents representative experiments (mean \pm S.E.M.); neurons pretreated with gp91 ds-tat (black trace) and neurons pretreated with scrambled gp91 ds-tat (grey trace). (f) Histogram summarizing the effect of gp91 ds-tat scrambled, NADPH oxidase assembly inhibitor gp91 ds-tat and inhibitor of Rac1 activator NSC-23766 on ROS changes during seizure-like activity. Error bars indicate S.E.M. ${ }^{* \star *} P<0.001$

depolarization in low-magnesium-treated neurons, supporting this as a significant confounder in previous studies (Figure 7b).

Apoptosis in the low magnesium model can be reduced by inhibiting ROS production. Excessive ROS production is known to lead to cell death. ${ }^{2}$ We therefore asked whether blocking sources of ROS generation might reduce cell death in the low magnesium model. The caspase-3 substrate NucView 488 allows measurements of apoptosis in real time. In time-series experiments with NucView 488, we observed apoptosis in a small fraction of neurons after $\sim 40 \mathrm{~min}$ in the low magnesium conditions (Figure $7 \mathrm{c}$ ). After $2 \mathrm{~h}, \sim 40 \%$ of neurons exhibited apoptosis. Pretreatment with inhibitor of NADPH oxidase assembly, gp91 ds-tat significantly reduced 


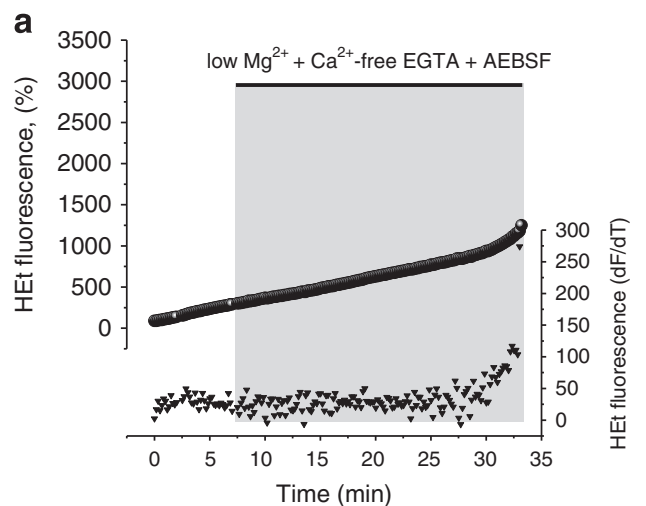

b

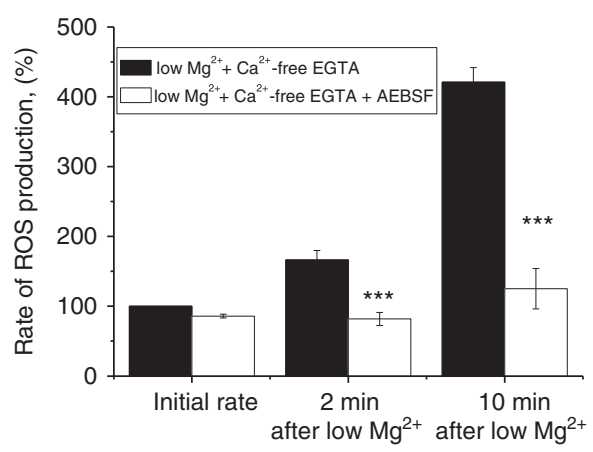

Figure 6 NADPH oxidase is the main source of ROS generation in neurons exposed to low magnesium $\mathrm{Ca}^{2+}{ }^{2}$-free EGTA: (a) HEt fluorescence measurements from a representative neuron under low $\mathrm{Mg}^{2+}$ - and $\mathrm{Ca}^{2+}$-free conditions when blocking NADPH oxidase with AEBSF $(20 \mu \mathrm{M}$; circles); bottom trace (triangles) indicates the trace after differentiation. (b) Histogram showing the mean ROS generation in neurons under low $\mathrm{Mg}^{2+}$ - and $\mathrm{Ca}^{2+}$-free conditions with (empty bars) and without AEBSF (black bars) at different time points. Error bars indicate S.E.M. ${ }^{* * *} P<0.001$

the proportion of neurons undergoing apoptosis in the low magnesium model of seizure-like activity (Figure $7 \mathrm{~d}$ ). To confirm this, we analysed the degree of apoptosis at $2 \mathrm{~h}$ in the different treatment groups. There was a significant difference in the rates of cell death between the four groups $(\mathrm{F}(3,11)=8.43$; one-way ANOVA; $P<0.01)$. Adding the NADPH oxidase inhibitor gp91 ds-tat or the $X O$ inhibitor oxypurinol to the low magnesium aCSF significantly reduced the percentage of apoptotic neurons when compared with low magnesium treatment alone $(P<0.05$; post hoc tests; Tukey's test; Figure 7e).

\section{Discussion}

Using live cell imaging, we have shown that seizure-like activity induces profound changes in the rate of ROS generation in neurons. This depends on the degree of neuronal activity. ROS generation was blocked with NMDA receptor antagonists, which also abolished the lowmagnesium-induced $\mathrm{Ca}^{2+}$ oscillations in neurons. To our surprise, low-magnesium-induced ROS generation was independent of external $\mathrm{Ca}^{2+}$, suggesting that a $\mathrm{Ca}^{2+}$-independent mechanism can trigger a cascade leading to an increase in neuronal ROS generation. Most importantly, the primary sources of ROS generation during neuronal hyperexcitability in this model were NADPH oxidase and $\mathrm{XO}$, whereas mitochondrial ROS generation, which was previously suggested to be the primary source of ROS generation during seizure-like activity, 8,35 did not contribute significantly to neuronal free radical load. Importantly, we also showed that targeting these mechanisms, by inhibiting NADPH oxidase or $\mathrm{XO}$ is neuroprotective.

Low-magnesium-induced ROS generation in neurons is dependent on NMDA receptor. We found that low magnesium-induced ROS generation is dependent on repetitive epileptiform activity, as it was not seen when the activity level of neurons was low as measured with simultaneous $\left[\mathrm{Ca}^{2+}\right]_{\mathrm{c}}$ recordings. Previous studies have found that low-magnesium-induced $\mathrm{Ca}^{2+}$ oscillations and the detrimental effect of NMDA receptor activation in cultures can be abolished by blocking NMDA receptors. ${ }^{29,36}$ In keeping with this, we were able to abolish ROS production in the low magnesium model of seizure-like activity by NMDA receptor antagonists. In addition, we were able to block ROS production and low-magnesium-induced $\mathrm{Ca}^{2+}$ oscillations by selectively blocking the NR2B subunit of the NMDA receptor, suggesting that ROS production is mainly mediated by the NR2B containing NMDA receptors. This is consistent with previous evidence that indicates that NMDA receptor excitotoxicity depends on the type of subunit expressed and on the location relative to the synapse, so that the, predominantly extrasynaptic, NR2B subunit containing NMDA receptors are responsible for the detrimental effects of NMDA receptor activation on neurons. ${ }^{37-40}$

NADPH oxidase is the primary source of free radical generation in the low magnesium model of epilepsy and can be activated independent of $\mathrm{Ca}^{2+}$ flux through the NMDA receptor. Previous studies have shown that the NMDA receptor-mediated ROS increase is linked to NADPH oxidase activation. ${ }^{16,17}$ However, these two reports have relied on excessive pharmacologic NMDA receptor activation (40 and $100 \mu \mathrm{M}$ NMDA), raise concerns about the physiologic and pathophysiologic relevance of this finding to disease. Our study, for the first time, provides evidence and mechanistic insight into free radical generation in an in vitro model of an acute neurologic disease. Our results convincingly show that NMDA-receptor-dependent ROS production in neurons is dependent on NADPH oxidase activation. NADPH oxidase in neurons can be activated through $\mathrm{Ca}^{2+}$-mediated activation of PKC, which in turn phosphorylates $\mathrm{p} 47^{\text {phox }}$ a subunit of the NADPH oxidase complex responsible for assembly with the NOX2 subunits. ${ }^{41}$ Interestingly, we found that NADPH oxidase was activated in low $\mathrm{Mg}^{2+}$ - and $\mathrm{Ca}^{2+}$-free conditions, implying a mechanism of NADPH oxidase activation that is independent of $\mathrm{Ca}^{2+}$ entry. Our results indicate that NOX2 may rely solely on $\mathrm{Na}^{+}$entry through NMDA receptors, something that has also been suggested in a study showing sodium-dependent activation of NOX2 in cardiomyocytes. ${ }^{42}$ Whether NADPH oxidase assembly relies on $\mathrm{Ca}^{2+}$-independent PKC activation in this setting remains to be determined. 
a
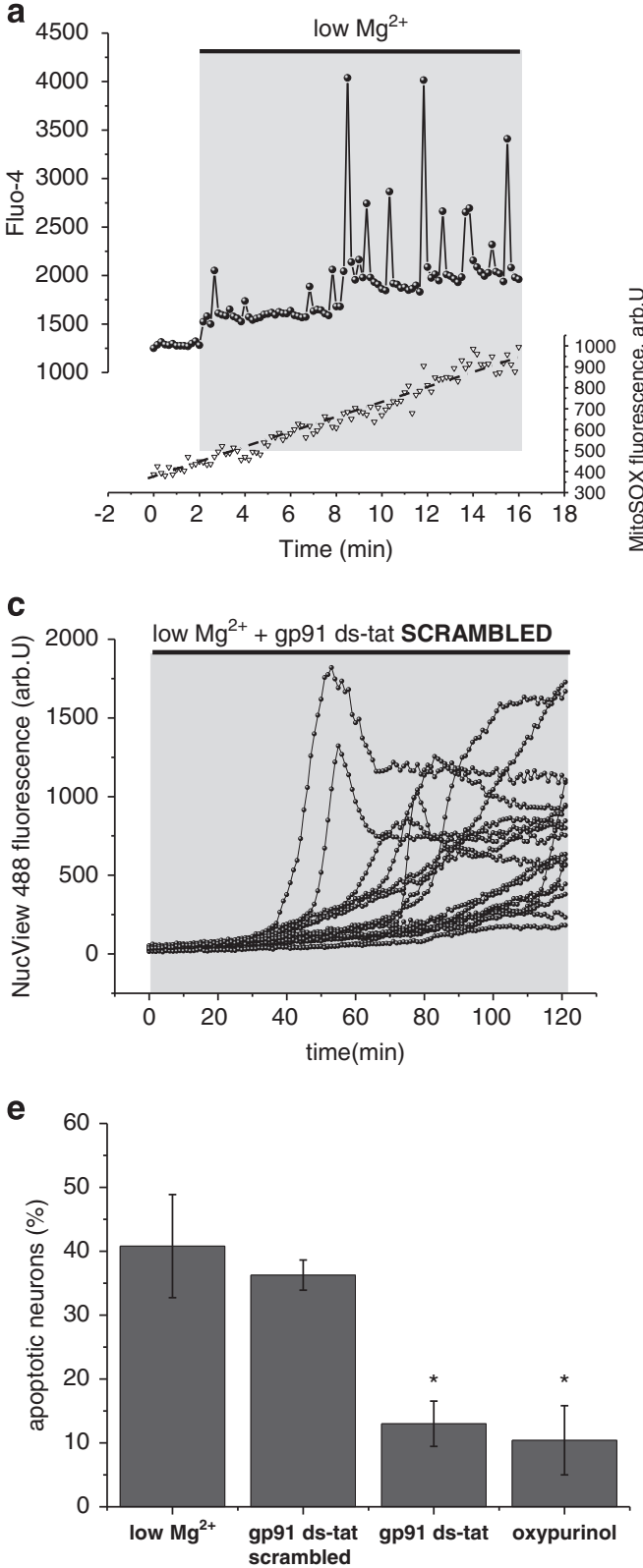

b

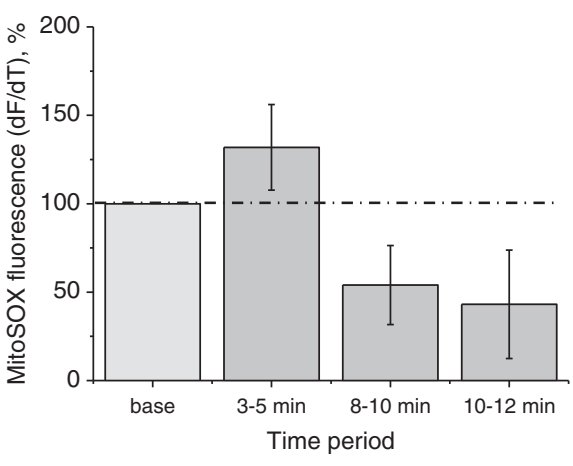

d

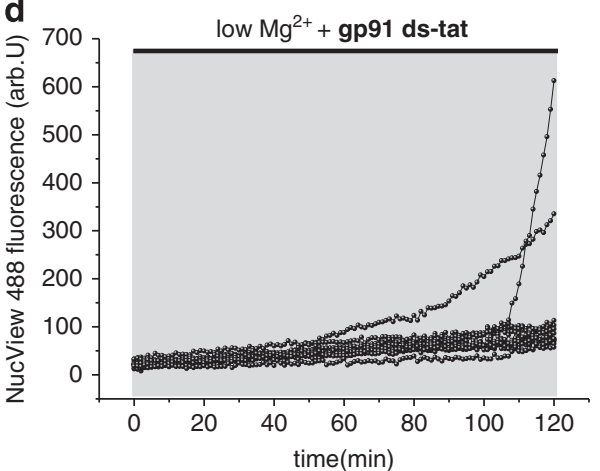

Figure 7 ROS of mitochondrial origin/NADPH oxidase and XO inhibition and apoptosis in vitro: (a) The upper trace shows a representative low-magnesium-induced [Ca $\left.{ }^{2+}\right]_{c}$ signal recorded using Fluo-4. The bottom trace shows the MitoSOX signal that was recorded simultaneously. (b) Histogram summarizing mean \pm S.E.M. rates of mitochondrial free radical generation in neurons at different time points. $(\mathbf{c}+\mathbf{d})$ Real-time measurements of apoptosis with NucView 488 . An increase in NucView 488 fluorescence indicates activation of caspase-3 and thus apoptosis. Each trace represents a single neuron. Note that within the low magnesium aCSF and (c) scrambled gp91 ds-tat peptide treatment group, first neurons undergo apoptosis after $~ 40$ min, whereas (d) inhibition of NADPH oxidase with gp91 ds-tat delays time to apoptosis. (e) Histogram summarizing mean \pm S.E.M. percentage of apoptotic neurons in different treatment groups. Error bars indicate S.E.M. ${ }^{*} P<0.05$ and ${ }^{* *} P<0.01$

This finding has important therapeutic implications as it suggests that NMDA-mediated detrimental ROS generation can be maintained without excessive calcium entry. Previous reports have shown that intracellular acidification of neurons can occur during transient NMDA receptor activation. ${ }^{43}$ We have extended this finding by showing that activation of NMDARs during low-magnesium-induced seizure-like activity is also sufficient to induce intracellular acidification.

Several in vivo and in vitro epilepsy models support the pivotal role of NADPH oxidase in seizures and epilepsy. In keeping with our findings, an increase in NADPH oxidase activity has been linked to chemoconvulsive epilepsy models such as the pilocarpine and kainate model of epilepsy. ${ }^{20,44}$ Inhibition of NADPH oxidase was effective in reducing cell death in the in vivo pilocarpine model of temporal lobe epilepsy. ${ }^{9,45}$ However, our study shows for the first time that the protective effect of NADPH oxidase inhibition is independent of chemoconvulsants.

XO contributes to a delayed increase in ROS generation in epileptiform activity. We identified that $X O$ inhibition resulted in decreased generation of ROS during 
low magnesium exposure. The inhibition of ROS generation affected the late phases (>10 min) of low magnesium exposure. These findings were difficult to capture in a group analysis as the onset of the late phase varied significantly between cells, indicating that the XO-dependent secondary ROS generation is not homogeneous. Intracellular ATP depletion occurs during continuous epileptiform activity, ${ }^{23,45}$ and results in adenine formation and consequently an increase in hypoxanthine and xanthine, substrates for $\mathrm{XO}^{31,32}$ Previous reports have shown a beneficial effect of $\mathrm{XO}$ inhibition on seizures. ${ }^{46-48}$ Our results confirm these observations, and for the first time provide evidence that inhibition of $\mathrm{XO}$ with oxypurinol reduces seizure-induced neuronal cell death.

Mitochondria are not the primary site of ROS generation in the low magnesium model of epilepsy. Increased free radical generation in seizures has commonly been ascribed to mitochondrial dysfunction; this assumption has, however, often been based on the coincidence of ROS changes and changes in mitochondrial metabolism during seizures. ${ }^{44,49}$ Despite studies suggesting that ROS of mitochondrial origin are the major source of ROS in epilepsy and specifically in the low magnesium model of epilepsy, ${ }^{8,35,50,51}$ we show here that ROS of mitochondrial origin are not the primary contributors to the neuronal free radical burden. Some of the previous work has only demonstrated indirect evidence of free radical formation through measures of glutathione, a major antioxidant. ${ }^{51,52} \mathrm{~A}$ major confounder in the interpretation of the few studies that use HEt to measure ROS production is that it is based on the temporal relationship between the increased HEt fluorescence and $\mathrm{Ca}^{2+}$ transients. However, HEt can undergo fluorescent dequenching on depolarization-induced release from the mitochondrial matrix to the cytoplasm. ${ }^{53}$ Using MitoSOX, a more specific mitochondrial ROS indicator, we were not able to support these previous findings, and, in contrast, observed a general decrease in mitochondrial ROS generation. Mild intracellular $\mathrm{Ca}^{2+}$ influx stimulates respiration and consequently leads to mitochondrial membrane potential hyperpolarization. This, in turn, can slightly increase the generation of ROS. ${ }^{54}$ However, mitochondrial membrane potential depolarization, as occurs during prolonged seizure-like events, ${ }^{14}$ inhibits mitochondrial ROS generation.

For the first time, we have provided a detailed insight into the importance of different ROS-generating pathways in hyperexcitability (summary in Figure 8). Our findings of ROS generation during hyperexcitability, the time course and the sources of ROS generation with a calcium- and mitochondriaindependent mechanism suggest novel interventional targets to prevent neuronal death in neurologic diseases associated with hyperexcitability, such as epilepsy, and challenge traditional views on exclusively mitochondria- and calciumdependent excitotoxicity.

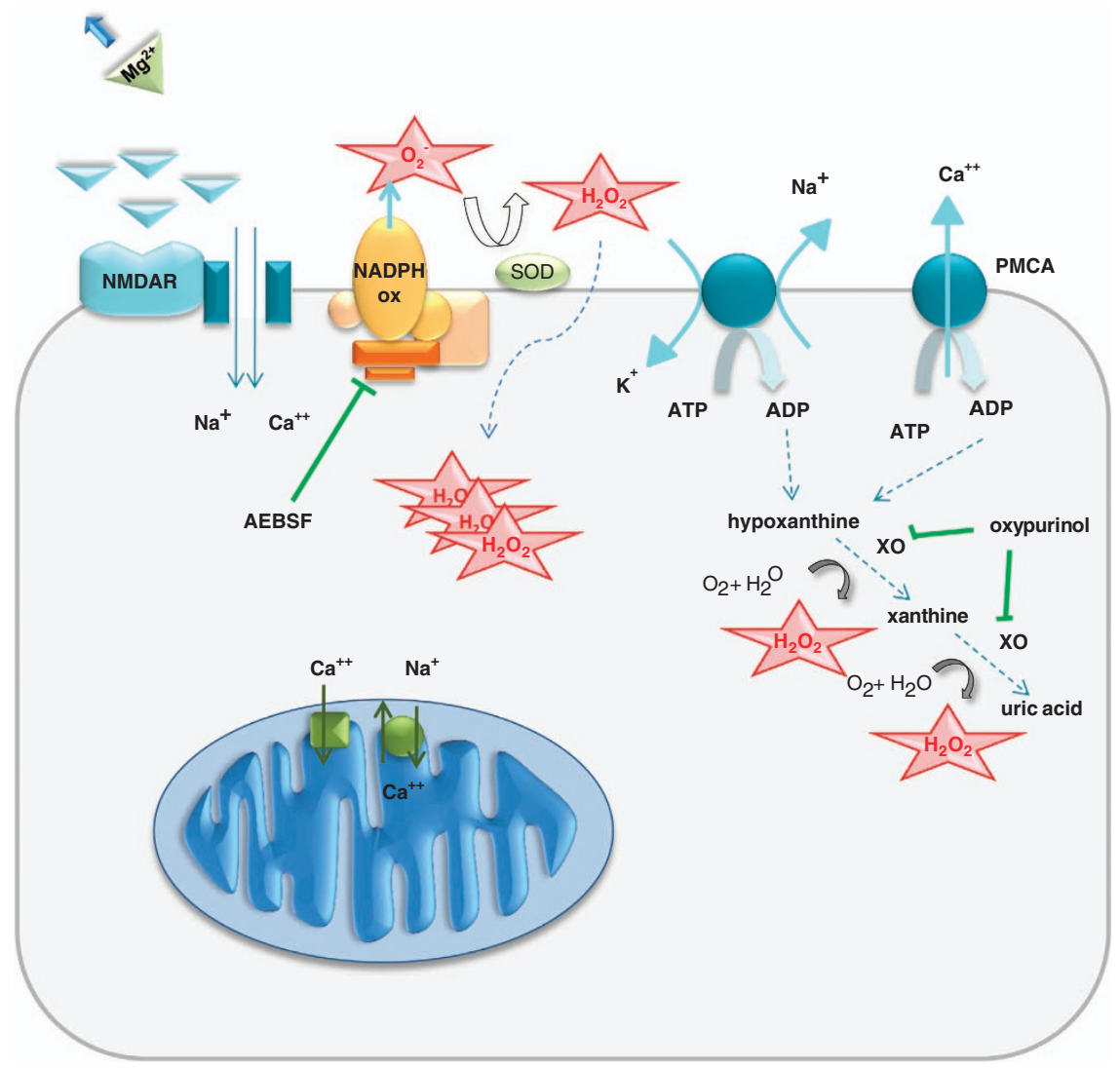

Figure 8 Schematic diagram illustrating the mechanisms of ROS generation in the low magnesium model of seizure-like activity: NADPH ox, NADPH oxidase; NMDAR, NMDA receptor; PMCA, plasma membrane $\mathrm{Ca}^{2+}$ ATPase; SOD, superoxide dismutase 
Materials and Methods

Cortical cell cultures. Mixed cocultures of cortical neurons and glial cells from postnatal (P0) Sprague-Dawley rats (UCL breeding colony) were prepared according to a modified protocol described by Haynes. ${ }^{55}$ Rat brains were quickly removed and neocortical tissue was cut and minced in ice-cold $\mathrm{HBSS}\left(\mathrm{Ca}^{2+}\right.$ and $\mathrm{Mg}^{2+}$ free; Gibco-Invitrogen, Paisley, UK). After treatment with $1 \%$ trypsin for $15 \mathrm{~min}$ at $37^{\circ} \mathrm{C}$ to dissociate the cells, residual trypsin was removed and the tissue was triturated. The suspension was plated onto poly-D-lysine/laminin-coated coverslips and cultured in Neurobasal A medium (Gibco-Invitrogen) supplemented with B-27 and $2 \mathrm{mM}$ L-glutamine (Gibco-Invitrogen). Experiments were carried out after 12-21 days in vitro to allow maturation of synapses in cultures. Neurons were identified and distinguished from glia by their smooth rounded somata and distinct processes using phase-contrast imaging.

Transfection with genetically encoded pH probe HyPer-C199S. At day 11, glioneuronal cultures were transfected with plasmid coding HyPer-C199S. HyPer-C199S, a pH indicator, has been recommended as an ideal control for the genetically encoded $\mathrm{H}_{2} \mathrm{O}_{2}$ indicator HyPer-3, as it is the $\mathrm{H}_{2} \mathrm{O}_{2}$ insensitive version of the HyPer-3. Transfections were performed using Effectene transfection reagent (Qiagen, Venlo, Netherlands) yielding $\sim 5 \%$ transfection efficiency in neurons. Cells were subjected to imaging, 1-3 days after transfection.

Imaging of intracellular ROS generation, $\left[\mathrm{Ca}^{2+}\right]_{c}, \mathrm{ROS}$ and $\left[\mathrm{Ca}^{2+}\right]_{\mathrm{c}}$ coregistration and $\left[\mathrm{Na}^{+}\right]_{\mathrm{c}}$. Fluorescent dyes were obtained from Invitrogen (Paisley, UK), unless otherwise stated. Preincubation and experimental procedures were performed at room temperature and all preincubations were performed in an HEPES-buffered salt solution (aCSF), composition in mM: 125 $\mathrm{NaCl}, 2.5 \mathrm{KCl}, 2 \mathrm{MgCl}_{2}, 1.25 \mathrm{KH}_{2} \mathrm{PO}_{4}, 2 \mathrm{CaCl}_{2}, 30$ glucose and $25 \mathrm{HEPES}, \mathrm{pH}$ adjusted to 7.4 with $\mathrm{NaOH}$. ROS generation was measured with $\mathrm{HEt}(2 \mu \mathrm{M}$, mostly superoxide) or $\mathrm{H}_{2} \mathrm{DFFDA}$ ( $20 \mu \mathrm{M}$, mostly hydrogen peroxide). To avoid accumulation of oxidized products, HEt was not preincubated, but was present in solutions throughout the experiments. In experiments using $\mathrm{H}_{2}$ DFFDA, cells were incubated for $30 \mathrm{~min}$ and washed before experiments. To target mitochondrial ROS production, cells were loaded with MitoSOX $(10 \mu \mathrm{M})$ for $10 \mathrm{~min}$ and washed before experiments. Intracellular $\mathrm{Ca}^{2+}$ measurements were performed using fura-2-AM (5 $\left.\mu \mathrm{M}\right)$ or Fluo-4 AM $(5 \mu \mathrm{M})$ and intracellular sodium levels were monitored with the ratiometric dye SBFI-AM $(10 \mu \mathrm{M}) . \mathrm{Ca}^{2+}$ and $\mathrm{Na}^{+}$indicators were loaded with $0.005 \%$ Pluronic $\mathrm{F}-127$ for $30 \mathrm{~min}$ and then washed. Rates of lipid peroxidation in neurons were measured using C11-BODIPY. Glioneuronal cocultures were preincubated for 20 min with C11-BODIPY (excitation 581/emission 591; $2 \mu \mathrm{M}$; Molecular Probes, Eugene, OR, USA). pH was monitored in neurons using carboxy-SNARF-AM $(10 \mu \mathrm{M})$, which were loaded with $0.005 \%$ Pluronic $\mathrm{F}-127$ for $30 \mathrm{~min}$ and then washed. To correlate the rate of ROS production to $\mathrm{Ca}^{2+}$ changes within the cell, ROS generation and $\left[\mathrm{Ca}^{2+}\right]_{\mathrm{c}}$ were measured simultaneously using HEt and Fluo-4 $\mathrm{AM}$. Experiments were carried out in either aCSF or excluding $\mathrm{MgCl}_{2}$ (low magnesium). Experiments with extracellular $\mathrm{Ca}^{2+}$ depletion were performed in aCSF omitting $\mathrm{Ca}^{2+}$ and adding the $\mathrm{Ca}^{2+}$ chelator EGTA $(0.5 \mathrm{mM})$.

Fluorescent images were obtained on an epifluorescence inverted microscope equipped with a $\times 20$ fluorite objective (Cairn Research, Kent, UK). Excitation wavelength was selected using a $10 \mathrm{~nm}$ bandpass filter centred on $340,380,490$ or $530 \mathrm{~nm}$ light as appropriate. Emitted fluorescence was detected by a cooled CCD camera after passing through a long-pass filter and 12-bit resolution. Laser power, gain and black level were optimized to obtain the full dynamic range while avoiding saturation for each excitation wavelength. Fluorescence of $\mathrm{H}_{2}$ DFFDA was excited by illumination at $490 \mathrm{~nm}$, whereas HEt was excited by illumination at $530 \mathrm{~nm}$. Ratiometric HEt fluorescence was recorded with excitation light at 380 and $530 \mathrm{~nm}$. For most of the experiments, we chose to perform measurements of ROS production rates with $\mathrm{HEt}$ at a single wavelength, first to avoid photobleaching and phototoxicity from excitation of cells in the range of UV light and second based on the fact that in some experiments $\left[\mathrm{Ca}^{2+}\right]_{\mathrm{c}}$ was coregistered with the ROS rate measurements. It was not possible to measure $\mathrm{H}_{2}$ DFFDA fluorescence alongside Fluo-4 fluorescence given the similar emission wavelength of these dyes. $\left[\mathrm{Ca}^{2+}\right]_{\mathrm{C}}$ and $\left[\mathrm{Na}^{+}\right]_{\mathrm{C}}$ were measured after exciting dyes with light provided by a xenon arc lamp, the beam passing through a monochromator at 340 and $380 \mathrm{~nm}$ with bandwidth of $10 \mathrm{~nm}$ (Cairn Research, Kent, UK). We presented traces as the ratio of excitation at 340 and $380 \mathrm{~nm}$, both with emission at $>515 \mathrm{~nm}$. Intracellular $\mathrm{Na}^{+}$and $\mathrm{Ca}^{2+}$ levels were expressed as ratios and were not calibrated to avoid inaccuracies arising from different calibration methods. Phototoxicity and photobleaching of cells was minimized by limiting light exposure to the time of acquisition of the images. Fluorescent images were acquired with a frame interval of $10 \mathrm{~s}$. Data were analysed using software from Andor (Belfast, UK). Illumination intensity was kept to a minimum (at $0.1-0.2 \%$ of laser output) to avoid phototoxicity and the pinhole set to give an optical slice of $\sim 2 \mu \mathrm{m}$. Rates of ROS increase were calculated at different time points (2 and $10 \mathrm{~min}$ ) after exposure to low magnesium aCSF only or to low magnesium aCSF in the presence of drug. These were compared with rates recorded during a 2-5 min aCSF exposure period referred to as baseline. Experiments were repeated at least four times using more than three different cultures.

All confocal images were obtained with a Zeiss 710 LSM (Jena, Germany) with an integrated META detection system. Coregistration of ROS production and $\mathrm{Ca}^{2+}$ signal was performed exciting HEt with the 565 laser and measuring light emitted at 580 $620 \mathrm{~nm}$. Simultaneous $\mathrm{Ca}^{2+}$ signals were acquired, exciting Fluo-4 with the $488 \mathrm{~nm}$ argon laser and measuring the emitted light at $500-550 \mathrm{~nm}$ (x40 objective). C11BODIPY (581/591) was excited using the 488 and $543 \mathrm{~nm}$ laser line and fluorescence measured using a bandpass filter from 505 to $550 \mathrm{~nm}$ and $560 \mathrm{~nm}$ long-pass filter (x40 objective). HyPer-C199S fluorescence was excited at 488 and $405 \mathrm{~nm}$ and emission set at $510-540 \mathrm{~nm}$ and expressed as 488/405 ratio. Carboxy-SNARF was excited using the $543 \mathrm{~nm}$ laser line and emission was collected at $580 \pm 30$ and $650 \pm 30 \mathrm{~nm}$. SNARF was expressed as $650 / 580$ ratio and was calibrated with brief application of $\mathrm{NH}_{4} \mathrm{Cl}_{2}$.

MitoSOX images were obtained using a $x 63$ objective to increase precision in measuring fluorescent signals immediately over mitochondria. MitoSOX were excited using the $565 \mathrm{~nm}$ laser line and fluorescence measured above $580 \mathrm{~nm}$. Compared with $\mathrm{HEt}$, MitoSOX is less potential sensitive and undergoes significantly less fluorescent dequenching. Fluorescent dequenching indicates the release of the dye to the cytosol and highlights the importance of measuring the signal immediately over mitochondria with high magnification as in our experimental set-up. ${ }^{5}$

Measuring apoptosis with NucView 488 caspase-3 substrate. Apoptosis was assessed both by measuring time series and time points within the experiment. NucView 488 caspase-3 substrate allows detection of caspase-3 activity in real time. Neuronal cultures were loaded for $15 \mathrm{~min}$ with $10 \mathrm{mM}$ NucView 488 caspase- 3 substrate (Biotium, Hayward, CA, USA). The $488 \mathrm{~nm}$ argon laser was used to excite NucView 488 fluorescence, which was measured using a bandpass filter from 510 and $560 \mathrm{~nm}$. Using phase-contrast optics, a bright-field image allowed identification of neurons, above the glial layer. ${ }^{5}$

Statistical analyses. Statistical analyses (two-tailed Student's t-test, one-way ANOVA, repeat-measure ANOVA, post hoc Tukey) were performed using SPSS 17.0 (SPSS, Chicago, IL, USA). Throughout ROS production was measured at two time points (2 and $10 \mathrm{~min}$ ) and a repeat-measures ANOVA was used for analysis with time at the within-subject factor. The significance level was set at $P<0.05$ and all data are given as mean \pm S.E.M.

\section{Conflict of Interest}

The authors declare no conflict of interest.

Acknowledgements. SK was supported by a stipend from the Deutsche Forschungsgemeinschaft (KO-3878/1-1). This work was supported by Epilepsy Research UK and FP7-HEALTH project 602102 (EPITARGET) We thank Dr Vsevolod V Belousov for kindly providing the genetically encoded probe HyPer-C199S.

1. Abramov AY, Canevari L, Duchen MR. Beta-amyloid peptides induce mitochondrial dysfunction and oxidative stress in astrocytes and death of neurons through activation of NADPH oxidase. J Neurosci 2004; 24: 565-575.

2. Chinta SJ, Andersen JK. Redox imbalance in Parkinson's disease. Biochim Biophys Acta 2008; 1780: 1362-1367.

3. Gandhi S, Wood-Kaczmar A, Yao Z, Plun-Favreau H, Deas E, Klupsch K et al. PINK1-associated Parkinson's disease is caused by neuronal vulnerability to calciuminduced cell death. Mol Cell 2009; 33: 627-638.

4. Halliwell B. Oxidative stress and neurodegeneration: where are we now? J Neurochem 2006; 97: 1634-1658.

5. Abramov AY, Scorziello A, Duchen MR. Three distinct mechanisms generate oxygen free radicals in neurons and contribute to cell death during anoxia and reoxygenation. $J$ Neurosci 2007; 27: 1129-1138

6. Slemmer JE, Shacka JJ, Sweeney MI, Weber JT. Antioxidants and free radical scavengers for the treatment of stroke, traumatic brain injury and aging. Curr Med Chem 2008; 15: 404-414. 
7. Frantseva MV, Velazquez JL, Hwang PA, Carlen PL. Free radical production correlates with cell death in an in vitro model of epilepsy. Eur J Neurosci 2000; 12: 1431-1439.

8. Malinska D, Kulawiak B, Kudin AP, Kovacs R, Huchzermeyer C, Kann O et al. Complex IIIdependent superoxide production of brain mitochondria contributes to seizure-related ROS formation. Biochim Biophys Acta 2010; 1797: 1163-1170.

9. Pestana RRF, Kinjo ER, Hernandes MS, Britto LRG. Reactive oxygen species generated by NADPH oxidase are involved in neurodegeneration in the pilocarpine model of temporal lobe epilepsy. Neurosci Lett 2010; 484: 187-191.

10. Waldbaum S, Patel M. Mitochondrial dysfunction and oxidative stress: a contributing link to acquired epilepsy?. J Bioenerg Biomembr 2010; 42: 449-455.

11. Tsai H-L, Chang C-N, Chang S-J. The effects of pilocarpine-induced status epilepticus on oxidative stress/damage in developing animals. Brain Dev 2010; 32: 25-31.

12. Layton ME, Pazdernik TL. Reactive oxidant species in piriform cortex extracellular fluid during seizures induced by systemic kainic acid in rats. J Mol Neurosci 1999; 13: 63-68.

13. Wang $X$, Fang H, Huang Z, Shang W, Hou T, Cheng A et al. Imaging ROS signaling in cells and animals. J Mol Med (Berl) 2013; 91: 917-927.

14. Kovács R, Kardos J, Heinemann U, Kann O. Mitochondrial calcium ion and membrane potential transients follow the pattern of epileptiform discharges in hippocampal slice cultures. J Neurosci 2005; 25: 4260-4269.

15. Schauwecker PE. Neuroprotection by glutamate receptor antagonists against seizureinduced excitotoxic cell death in the aging brain. Exp Neurol 2010; 224: 207-218.

16. Girouard H, Wang G, Gallo EF, Anrather J, Zhou P, Pickel VM et al. NMDA receptor activation increases free radical production through nitric oxide and NOX2. J Neurosci 2009; 29: 2545-2552.

17. Brennan AM, Suh SW, Won SJ, Narasimhan P, Kauppinen TM, Lee H et al. NADPH oxidase is the primary source of superoxide induced by NMDA receptor activation. Nat Neurosci 2009; 12: 857-863.

18. Schiavone S, Jaquet V, Sorce S, Dubois-Dauphin M, Hultqvist M, Bäckdahl L et al. NADPH oxidase elevations in pyramidal neurons drive psychosocial stress-induced neuropathology. Transl Psychiatry 2012; 2: e111.

19. Cristóvão AC, Guhathakurta S, Bok E, Je G, Yoo SD, Choi D-H et al. NADPH oxidase 1 mediates $\alpha$-synucleinopathy in Parkinson's disease. J Neurosci 2012; 32: 14465-14477.

20. Di Maio R, Mastroberardino PG, Hu X, Montero L, Greenamyre JT. Pilocapine alters NMDA receptor expression and function in hippocampal neurons: NADPH oxidase and ERK1/2 mechanisms. Neurobiol Dis 2011; 42: 482-495.

21. DeLorenzo RJ, Pal S, Sombati S. Prolonged activation of the $\mathrm{N}$-methyl-D-aspartate receptor-Ca2+ transduction pathway causes spontaneous recurrent epileptiform discharges in hippocampal neurons in culture. Proc Natl Acad Sci USA 1998; 95: 14482-14487.

22. Blair RE, Deshpande LS, Sombati S, Elphick MR, Martin BR, DeLorenzo RJ. Prolonged exposure to WIN55,212-2 causes downregulation of the CB1 receptor and the development of tolerance to its anticonvulsant effects in the hippocampal neuronal culture model of acquired epilepsy. Neuropharmacology 2009; 57: 208-218.

23. Kovac S, Domijan A-M, Walker MC, Abramov AY. Prolonged seizure activity impairs mitochondrial bioenergetics and induces cell death. J Cell Sci. 2012; 125(Part 7): 1796-1806.

24. Kovacs R, Kardos J, Heinemann U, Kann O. Mitochondrial calcium ion and membrane potential transients follow the pattern of epileptiform discharges in hippocampal slice cultures. J Neurosci 2005; 25: 4260-4269.

25. Kovacs R, Schuchmann S, Gabriel S, Kardos J, Heinemann U. Ca ${ }^{2+}$ signalling and changes of mitochondrial function during low- $\mathrm{Mg}^{2+}$-induced epileptiform activity in organotypic hippocampal slice cultures. Eur J Neurosci 2001; 13: 1311-1319.

26. Barbarosie M, Avoli M. CA3-driven hippocampal-entorhinal loop controls rather than sustains in vitro limbic seizures. J Neurosci 1997; 17: 9308-9314.

27. Barkai E, Friedman A, Grossman Y, Gutnick MJ. Laminar pattern of synaptic inhibition during convulsive activity induced by 4-aminopyridine in neocortical slices. J Neurophysiol 1995; 73: $1462-1467$.

28. Sun DA, Sombati S, DeLorenzo RJ. Glutamate injury-induced epileptogenesis in hippocampal neurons: an in vitro model of stroke-induced 'epilepsy'. Stroke 2001; 32: 2344-2350.

29. DeLorenzo RJ, Sun DA, Deshpande LS. Cellular mechanisms underlying acquired epilepsy: the calcium hypothesis of the induction and maintainance of epilepsy. Pharmacol Ther 2005 105: 229.

30. Diatchuk V, Lotan O, Koshkin V, Wikstroem P, Pick E. Inhibition of NADPH oxidase activation by 4-(2-aminoethyl)-benzenesulfonyl fluoride and related compounds. J Biol Chem 1997; 272: 13292-13301.

31. Kinugasa $Y$, Ogino K, Furuse Y, Shiomi T, Tsutsui H, Yamamoto T et al. Allopurinol improves cardiac dysfunction after ischemia-reperfusion via reduction of oxidative stress in isolated perfused rat hearts. Circ J 2003; 67: 781-787.
32. Xia Y, Zweier JL. Substrate control of free radical generation from xanthine oxidase in the postischemic heart. J Biol Chem 1995; 270: 18797-18803.

33. Rey FE, Cifuentes ME, Kiarash A, Quinn MT, Pagano PJ. Novel competitive inhibitor of NAD (P) $\mathrm{H}$ oxidase assembly attenuates vascular $\mathrm{O}(2)(-)$ and systolic blood pressure in mice. Circ Res 2001; 89: 408-414.

34. Elnakish MT, Hassanain HH, Janssen PM, Angelos MG, Khan M. Emerging role of oxidative stress in metabolic syndrome and cardiovascular diseases: important role of Rac/NADPH oxidase. J Pathol 2013; 231: 290-300.

35. Kovács R, Schuchmann S, Gabriel S, Kann O, Kardos J, Heinemann U. Free radicalmediated cell damage after experimental status epilepticus in hippocampal slice cultures. J Neurophysiol 2002; 88: 2909-2918.

36. Mangan PS, Kapur J. Factors underlying bursting behavior in a network of cultured hippocampal neurons exposed to zero magnesium. J Neurophysiol 2004; 91: 946-957.

37. Hardingham GE, Fukunaga $Y$, Bading H. Extrasynaptic NMDARs oppose synaptic NMDARs by triggering CREB shut-off and cell death pathways. Nat Neurosci 2002; 5: 405-414.

38. Liu F-Y, Wang X-F, Li M-W, Li J-M, Xi Z-Q, Luan G-M et al. Upregulated expression of postsynaptic density- 93 and $N$-methyl-D-aspartate receptors subunits $2 B$ mRNA in temporal lobe tissue of epilepsy. Biochem Biophys Res Commun 2007; 358: 825-830.

39. Monyer H, Burnashev N, Laurie DJ, Sakmann B, Seeburg PH. Developmental and regional expression in the rat brain and functional properties of four NMDA receptors. Neuron 1994; 12: $529-540$.

40. Tovar KR, Westbrook GL. The incorporation of NMDA receptors with a distinct subunit composition at nascent hippocampal synapses in vitro. J Neurosci 1999; 19: 4180-4188.

41. Bedard K, Krause K-H. The NOX family of ROS-generating NADPH oxidases: physiology and pathophysiology. Physiol Rev 2007; 87: 245-313.

42. Balteau M, Tajeddine N, de Meester C, Ginion A, Des Rosiers C, Brady NR et al. NADPH oxidase activation by hyperglycaemia in cardiomyocytes is independent of glucose metabolism but requires SGLT1. Cardiovasc Res 2011; 92: 237-246.

43. Rathje M, Fang H, Bachman JL, Anggono V, Gether U, Huganir RL et al. AMPA receptor pHluorin-GluA2 reports NMDA receptor-induced intracellular acidification in hippocampal neurons. Proc Natl Acad Sci USA 2013; 110: 14426-14431.

44. Patel M, Li Q-Y, Chang L-Y, Crapo J, Liang L-P. Activation of NADPH oxidase and extracellular superoxide production in seizure-induced hippocampal damage. $J$ Neurochem 2005; 92: 123-131.

45. Kim JH, Jang BG, Choi BY, Kim HS, Sohn M, Chung TN et al. Post-treatment of an NADPH oxidase inhibitor prevents seizure-induced neuronal death. Brain Res 2013; 1499: 163-172.

46. Kramer LD, Locke GE, Nelson LG, Ogunyemi AO. Status epilepticus following withdrawal of allopurinol. Ann Neurol 1990; 27: 691.

47. Tada H, Morooka K, Arimoto K, Matsuo T. Clinical effects of allopurinol on intractable epilepsy. Epilepsia 1991; 32: 279-283.

48. Zagnoni PG, Bianchi A, Zolo P, Canger R, Cornaggia C, D'Alessandro P et al. Allopurinol as add-on therapy in refractory epilepsy: a double-blind placebo-controlled randomized study. Epilepsia 1994; 35: 107-112.

49. Cock H. The role of mitochondria in status epilepticus. Epilepsia 2007; 48: 24-27.

50. Liang L-P, Patel M. Mitochondrial oxidative stress and increased seizure susceptibility in Sod2 (-/+) mice. Free Radic Biol Med 2004; 36(Suppl 8): 542-554.

51. Liang L-P, Patel M. Seizure-induced changes in mitochondrial redox status. Free Radic Biol Med 2006; 40: 316-322.

52. Cock HR, Tong X, Hargreaves IP, Heales SJR, Clark JB, Patsalos PN et al. Mitochondrial dysfunction associated with neuronal death following status epilepticus in rat. Epilepsy Res 2002; 48: 157-168.

53. Budd SL, Castilho RF, Nicholls DG. Mitochondrial membrane potential and hydroethidinemonitored superoxide generation in cultured cerebellar granule cells. FEBS Lett 1997; 415: $21-24$.

54. Nicholls DG. Mitochondria and calcium signaling. Cell Calcium 2005; 38: 311-317.

55. Haynes LW. The Neuron in Tissue Culture. New Yok, NY, USA: Wiley, 1999.

(c) Cell Death and Disease is an open-access journal published by Nature Publishing Group. This work is licensed under a Creative Commons Attribution 3.0 Unported License. The images or other third party material in this article are included in the article's Creative Commons license, unless indicated otherwise in the credit line; if the material is not included under the Creative Commons license, users will need to obtain permission from the license holder to reproduce the material. To view a copy of this license, visit http:// creativecommons.org/licenses/by/3.0/ 